\title{
Fermilab
}

Managed by Fermi Research Alliance, LLC for the U.S. Department of Energy Office of Science

\section{RCS for Multi-MW Facility at Fermilab}

\author{
Jeffrey Eldred \\ MW Rings Workshop at Fermilab \\ May 2018
}

This document was prepared by [DUNE Collaboration] using the resources of the Fermi National Accelerator Laboratory (Fermilab), a U.S. Department of Energy, Office of Science, HEP User Facility. Fermilab is managed by Fermi Research Alliance, LLC (FRA), acting under Contract No. DEAC02-07CH11359 


\section{Fermilab Proton Accelerator Facility}

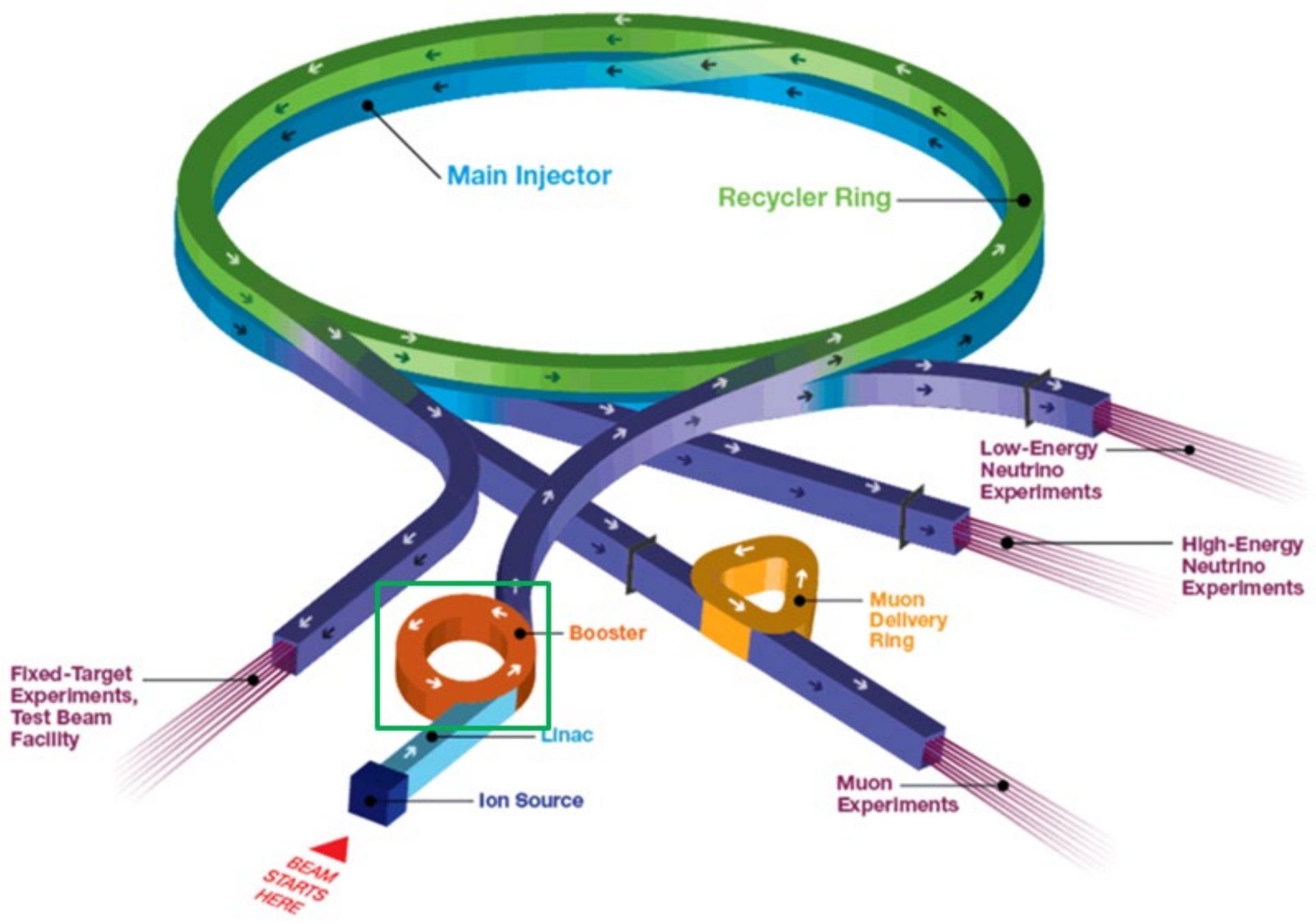

\section{荤 Fermilab}

2 Jeffrey Eldred |RCS for Multi-MW Facility at Fermilab

$6 / 4 / 2019$ 


\section{Booster Performance}

Booster improvement is an ongoing effort Yesterday's talks provide a good overview.

\section{Known challenges include:}

1. Transition crossing.

2. Impedance effects from dipole laminations.

3. Space-charge forces at injection.

4. Lattice optics correction. 


\section{New RCS for Multi-MW Facility}




\section{(Proposed) RCS Intensity Upgrade}

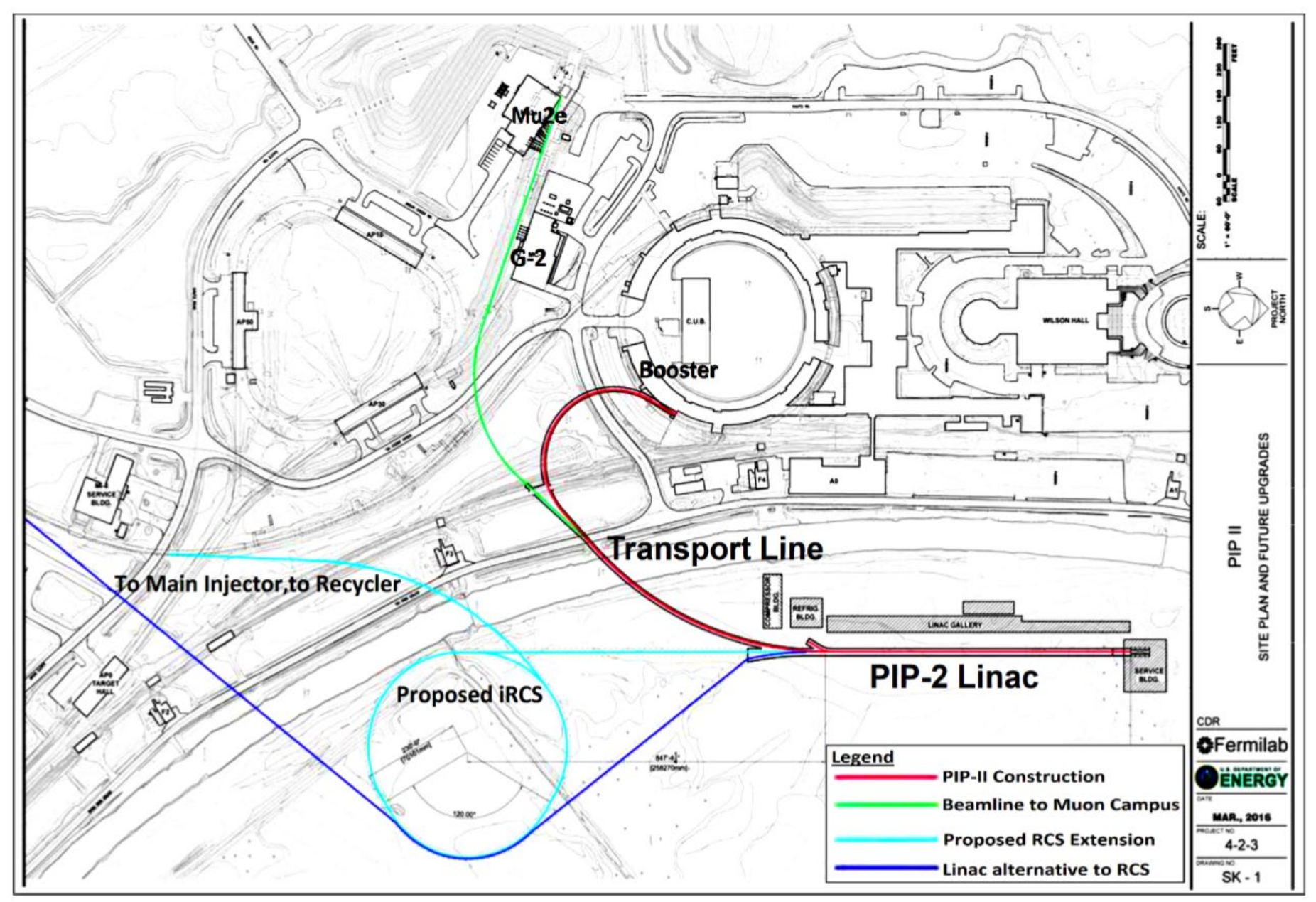




\section{Benefits of Modern RCS Design}

\section{Eliminate Transition Crossing}

Lattice Improvements for Injection Intensity

- Higher periodicity, for suppression of harmonic resonances.

- Lower maximum beta functions, for greater beam acceptance.

- Well-characterized separate-function magnets, for better optics.

Other Improvements

- Reduce sources of impedance.

- Dispersion-free RF acceleration.

- Perpendicular-bias RF cavities.

- Low-SEY coating for mitigation of electron cloud. 


\section{Basic Scenario - Intense Boxcar Stacking}

\section{PIP-II for $1.2 \mathrm{MW}$ at $120 \mathrm{GeV}$ :}

- Booster intensity of $6.5 \mathrm{e} 12$ with $20 \mathrm{~Hz}$ ramp-rate.

- Slip-stacking in Recycler.

- 12 batches in Main Injector with 1.2 sec ramp.

RCS for $2.4 \mathrm{MW}$ at $120 \mathrm{GeV}$ :

- RCS intensity of $36 \mathrm{e} 12$ with $20 \mathrm{~Hz}$ ramp-rate.

- Boxcar stacking in Recycler (no slip-stacking)

- 5 batches in Main Injector with 1.4 sec ramp.

To achieve 2.4 MW, we need to quadruple the linear charge density.

If we can do that, an RCS opens options for even higher power. 


\section{Laslett Tune Shift}

Laslett tune-shift:

$$
\Delta \nu \approx \frac{N r_{0}}{2 \pi \epsilon_{N} \beta \gamma^{2}} F B
$$

Space-Charge Limit:

$$
\begin{gathered}
N \sim \epsilon_{N} \times \beta \gamma^{2} \times \Delta \nu_{\max } \\
v=6.5 \text { half-integer } \\
\text { resonance constrains } \\
\text { Booster to } 0.3-0.35
\end{gathered}
$$

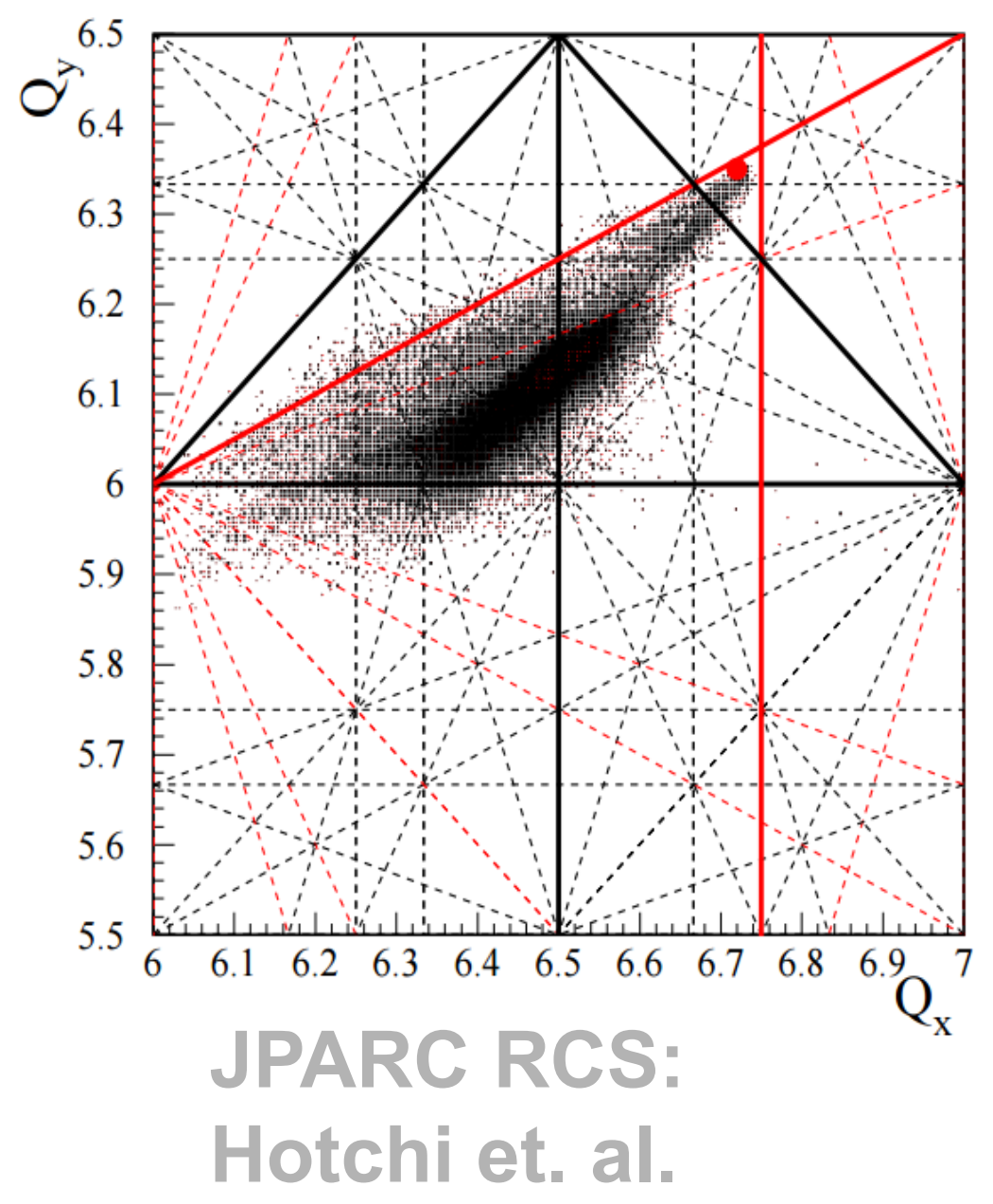

Fermilab 


\section{Aperture and Emittance}

The vertical gap in the Booster is $5.72 \mathrm{~cm}(2.25$ ") at the location where $\beta_{y}=35 \mathrm{~m}$.

$$
y_{\max }=\sqrt{\left(\frac{\epsilon_{N, \max }}{\beta \gamma}\right) \beta_{y, \max }}
$$

This determines the Booster $95 \%$ normalized emittance of $15 \mathrm{~mm}$ mrad.

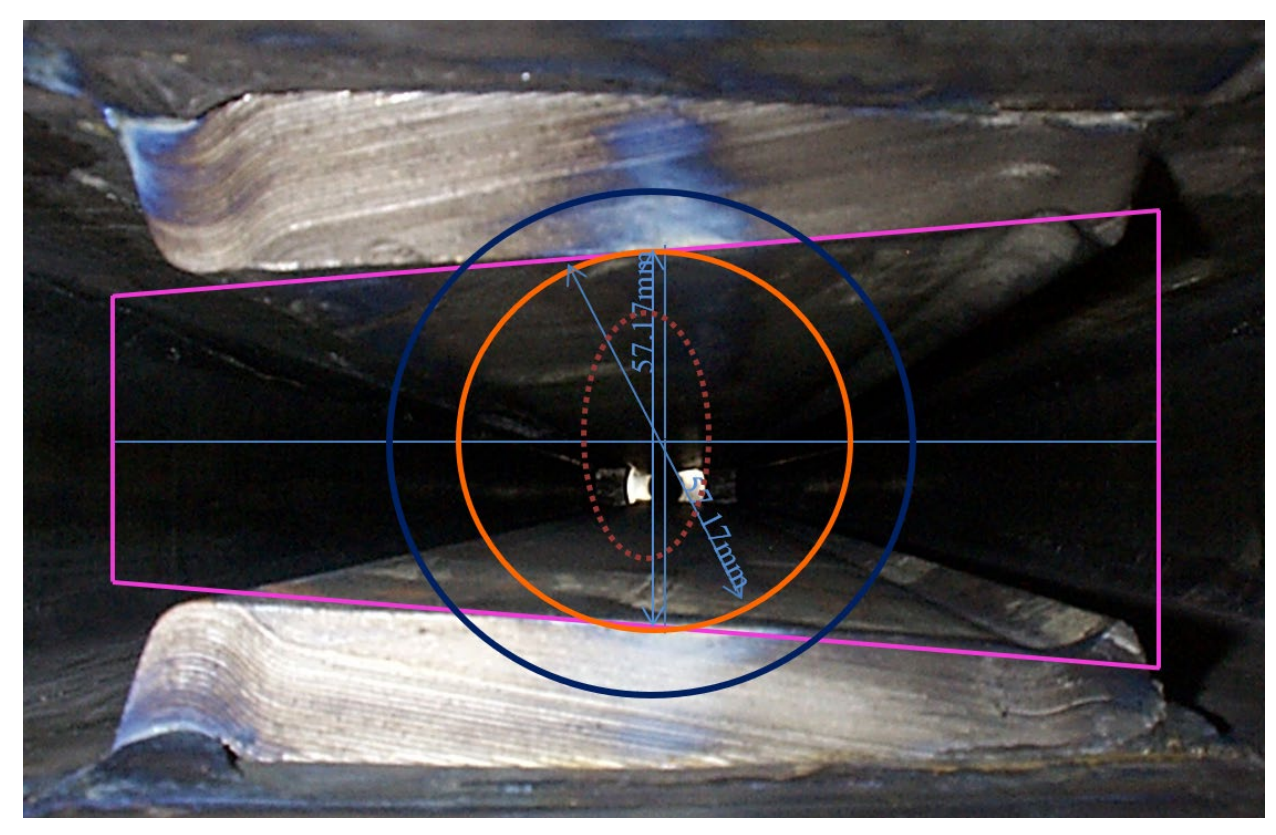

\section{T.K. Kroc}

An RCS with smaller betas or higher injection energy can reach a $95 \%$ normalized emittance of $\mathbf{3 0 ~} \mathbf{~ m m ~} \mathbf{m r a d}$ at Space-Charge Limit:

$$
N \sim \epsilon_{N} \times \beta \gamma^{2} \times \Delta \nu_{\max }
$$
the same aperture. 


\section{Baseline RCS Lattice}

Simple FODO Lattice

Avoids Transition

Dispersion-free Arcs

Low Max Beta

Circumference 553 m

Backup slides give additional details.

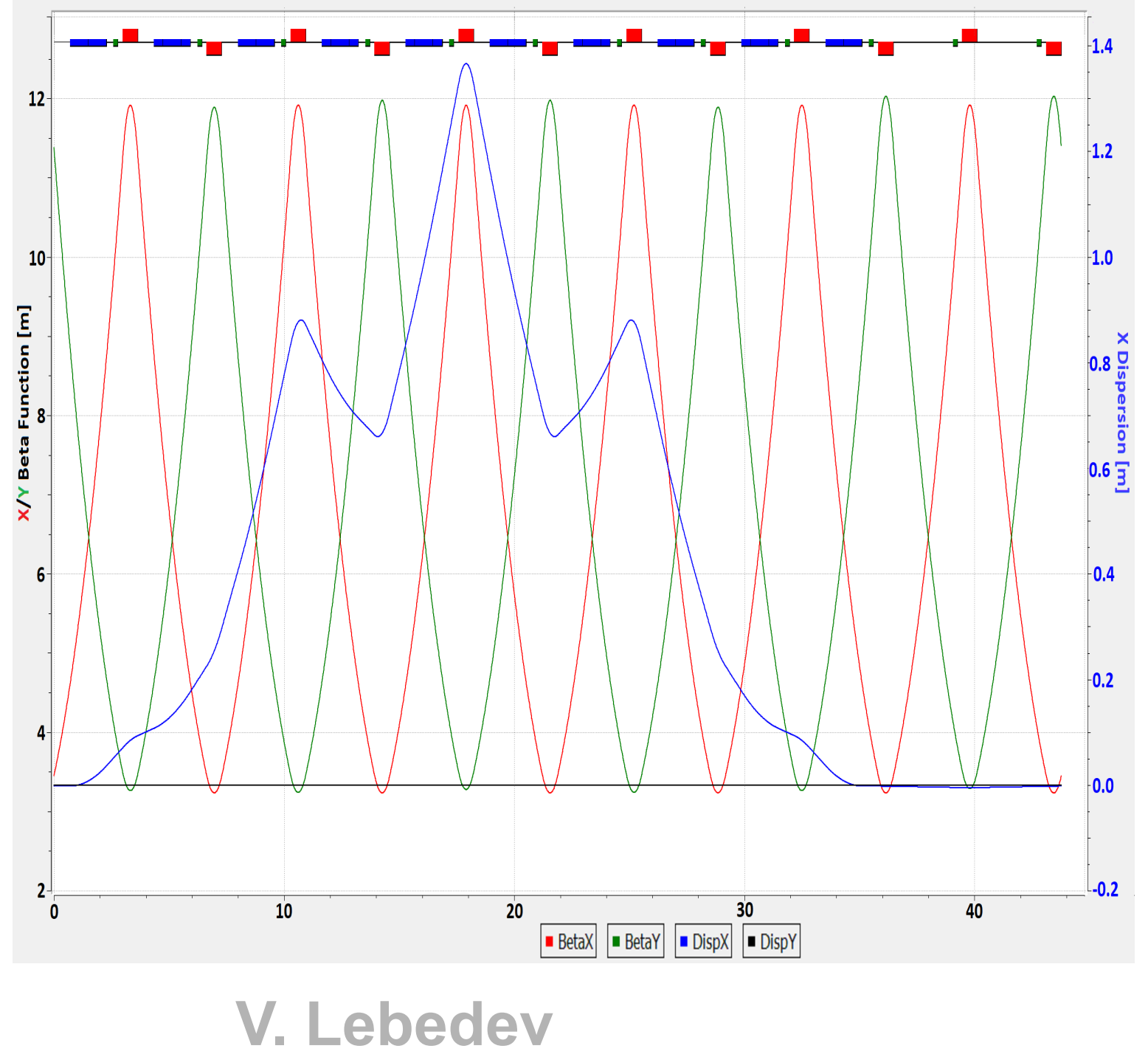

茶Fermilab 


\section{J-PARC RCS as Precedent}

The J-PARC RCS shows intensity of 83e12 protons, $1 \mathrm{MW}$ extracted beam power, $\mathbf{0 . 3 0}$ tune-shift. Hotchi et al. PRAB 2017.

This design has a large aperture (x12 Booster) and goes to $3 \mathrm{GeV}$. Our ring needs higher energy and cannot benefit from that aperture.

\section{Ring Scaling Exercise:}

- Scale up injection energy, scale up circumference, scale down aperture, scale down max beta, scale up bunching factor

- Keep the ratios between beampipe acceptance, collimator acceptance, and geometric emittance fixed. Keep maximum space-charge tune-shift fixed.

- Extraction energy $10 \mathrm{GeV}$, normalized emittance is $\mathbf{3 0} \mathbf{~ m m ~ m r a d ,}$ dipole gap $5.4 \mathrm{~cm}$, beam intensity $\mathbf{3 6 e 1 2}$, tune-shift $\mathbf{0 . 3 0}$. 


\section{Eddy Currents in Vacuum Chamber}

Vacuum chamber radius a: $2.8 \mathrm{~cm}$.

Ramp rate: $20 \mathrm{~Hz}$

Vacuum chamber heating power by eddy currents

$$
\frac{d P}{d z}=\frac{\pi \sigma_{r} d a^{3}}{2 c^{2}} \omega^{2} B_{A C}^{2}
$$

1.28mm Steel-316: $108 \mathrm{~W} / \mathrm{m}$

0.75mm Inconel-625: $36 \mathrm{~W} / \mathrm{m}$

Conservative air cooling estimate of convective cooling heat transfer coefficient $10^{-3} \mathrm{~W} / \mathrm{cm}^{2} / \mathrm{K}$

1.28mm Steel-316: $\Delta T=60 \mathrm{~K}$

0.75mm Inconel-625: $\Delta \mathrm{T}=20 \mathrm{~K}$ 


\section{Integrable RCS Design}

An RCS based on demonstrated design principles can reach the performance we need based to achieve 2.4 MW at Fermilab.

The RCS upgrade scenario is not contingent on integrable design.

But integrable RCS lattice designs are worth studying. Strong nonlinear focusing offers a way to suppress halo formation and enhance Landau damping. 


\section{Integrable RCS Lattice design}

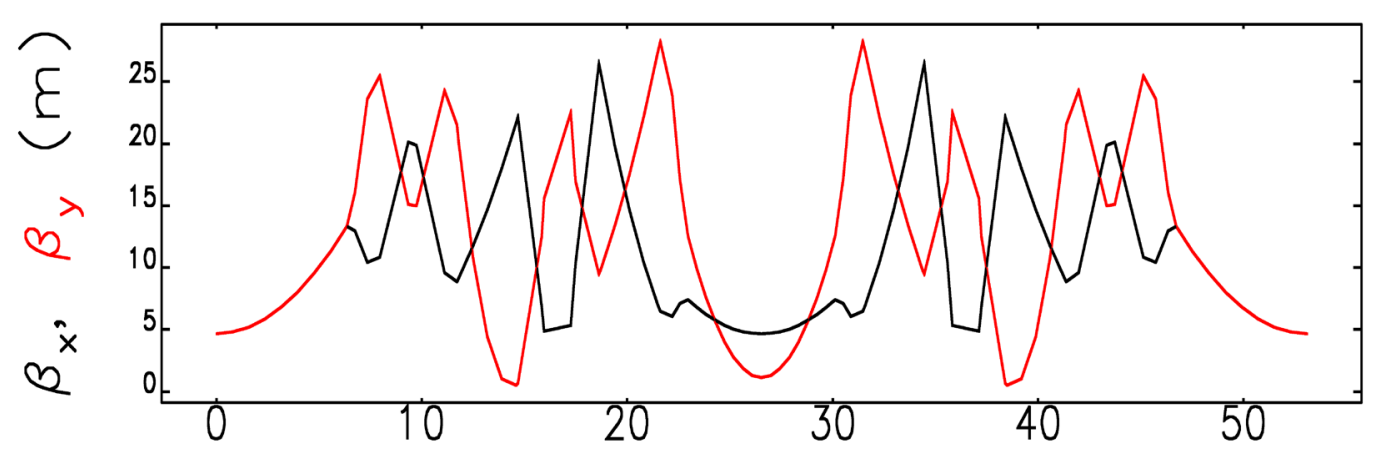

Periodicity: 12

Circumference: $636 \mathrm{~m}$

Bend-radius rho: $15.4 \mathrm{~m}$

Max Beta $x, y$ function: 25 m

Max Dispersion function: $\mathbf{0 . 2 2} \mathbf{~ m}$

RF Insertion length: $7.2 \mathrm{~m}, 4 \times 1.3 \mathrm{~m}$

NL Insertion length: $\mathbf{1 2 . 7} \mathrm{m}$

Insert Phase-Advance: $\mathbf{0 . 4}$

Minimum c-value: $\mathbf{3} \mathbf{~ c m}$

Beta at insert center: $\mathbf{5} \mathbf{~ m}$

Betatron Tune: $\mathbf{2 1 . 6}$

Natural Chromaticity: $\mathbf{- 7 9}$

Second-order Chromaticity: 1600

Synchrotron Tune: $\mathbf{0 . 0 8}$

${ }_{s(m)}^{30} \stackrel{40}{ }{ }^{40}$ Eldred, Valishev IPAC 2018 


\section{Space-charge Simulation of iRCS}

Beam injected with $20 \%$ mismatch

Laslett tune-shift of 0.4 , corresponding to $32 \mathrm{e} 12$ protons. Eldred, Beam stable 5000 revolutions, halo strongly suppressed. Valishev

Caveat: Perfect lattice with no errors.
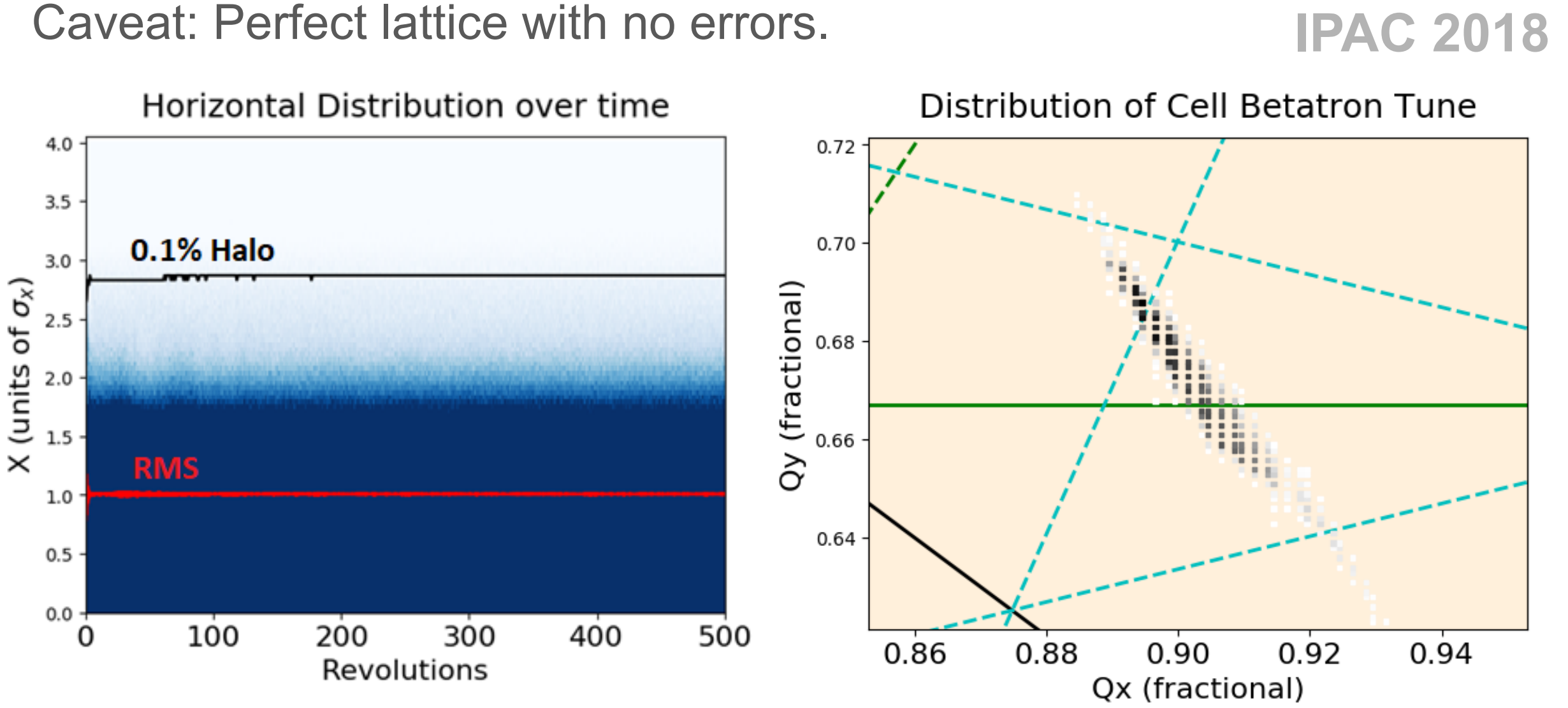

15 Jeffrey Eldred |RCS for Multi-MW Facility at Fermilab 


\section{Implications for Linac, Recycler and Main Injector}




\section{PIP-II Linac for new RCS}

\section{PIP-II Linac}

- MEBT chops $5 \mathrm{~mA}$ RFQ current to $2 \mathrm{~mA}$,

- chops two out of five $650 \mathrm{MHz}$ bunches.

- Delivers 2 mA current every $20 \mathrm{~Hz}$ for $\mathbf{0 . 6}$ ms.

\section{Linac Intensity Upgrade}

- RCS intensity of $36 \mathrm{e} 12$ with $20 \mathrm{~Hz}$ ramp-rate.

- Fill time with 5 mA current every $20 \mathrm{~Hz}$ for $1.3 \mathrm{~ms}$.

- Preferable to $2 \mathrm{~mA}$ current for $3 \mathrm{~ms}$.

- This fill rate requires a power-amplifier upgrade to PIP-II Linac. 


\section{Boxcar Stacking from Booster and Future RCS}

- Booster Circumference $\mathbf{4 7 4}$ m, 84 buckets.

- MI/RR Circumference $\mathbf{3 3 1 8 ~ m , ~ 7 x 8 4 ~ b u c k e t s . ~}$

- Ml extraction kicker gap, 24 buckets

Assume the same integrated dipole field per length as the Booster, but consider a larger circumference:

\begin{tabular}{|l|l|l|}
\hline Circumference & N Batches & Max Energy \\
\hline $474-530 \mathrm{~m}$ & 6 & $8.0-9.0 \mathrm{GeV}$ \\
\hline $569-656 \mathrm{~m}$ & 5 & $9.6-10.7 \mathrm{GeV}$ \\
\hline $711-796 \mathrm{~m}$ & 4 & $12.0-13.4 \mathrm{GeV}$ \\
\hline
\end{tabular}




\section{Should we use the Recycler?}

\section{8-GeV stacking with Recycler}

- 5 batches injected at $20-\mathrm{Hz}$ requires 0.2 seconds.

- Using the Recycler for accumulation, Ml cycle time goes from $1.4 \mathrm{~s}$. to $1.2 \mathrm{~s}$, for a $\mathbf{1 . 1 7}$ factor improvement in beam power.

\section{0-GeV MI Injection}

- Laslett tune-spread reduced by factor $\mathbf{1 . 5 0}$ at injection.

- Beam size reduced by factor 1.22 at injection.

- Incompatible with 8-GeV Recycler.

The RCS should be capable of $10-\mathrm{GeV}$

- We can keep both options open 


\section{Recycler Intensity Limits}

\section{Space-charge Tune-spread Losses:}

Current Tune-Spread

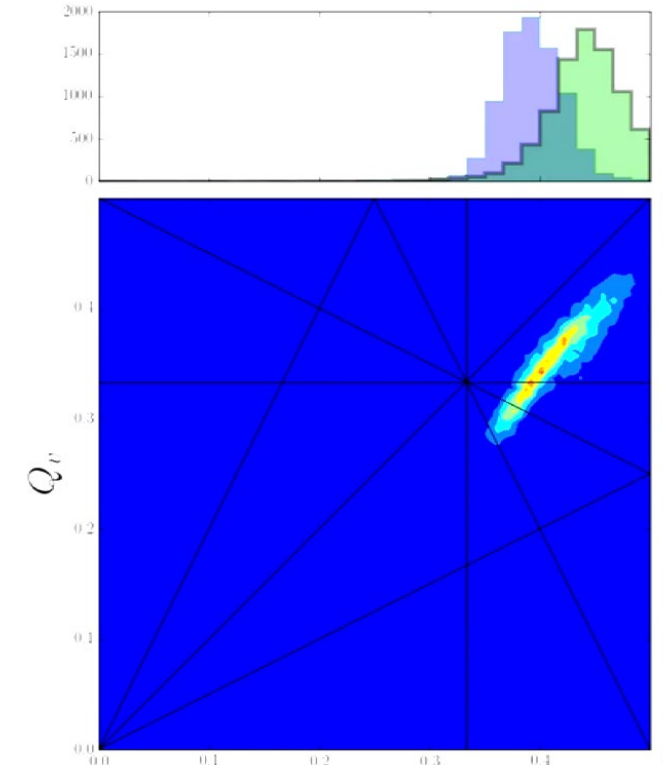

$Q_{h}$
PIP-II Tune-Spread

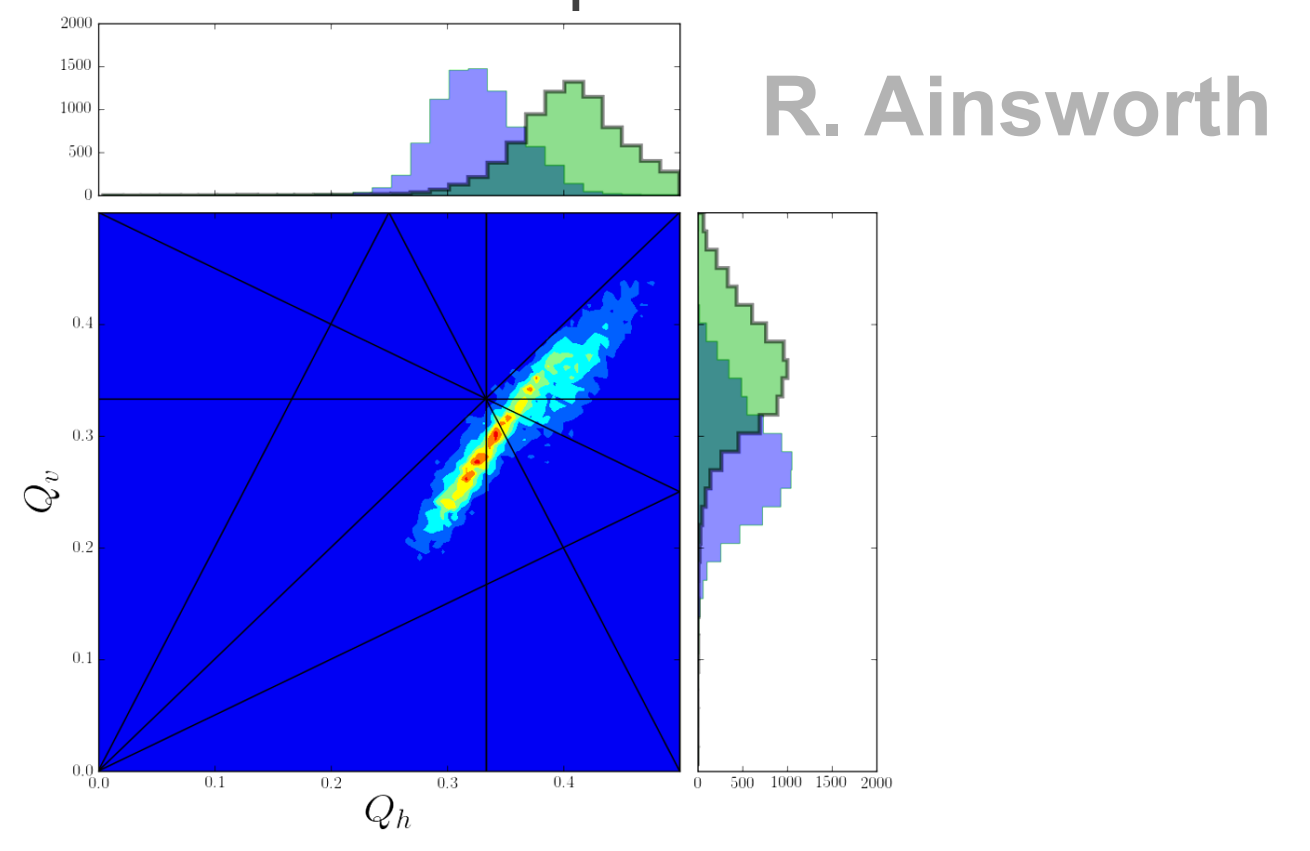

If we go to higher than PIP-II intensity, but without a momentum separation between the beams, we will cross the same res. lines.

How well can we compensate the resonances lines? 


\section{Recycler Intensity Limits}

\section{Tight Aperture Losses:}

- For PIP-II era, smaller cleaner beam.

- For RCS, limited by RR/MI apertures.
Main Injector beam pipe

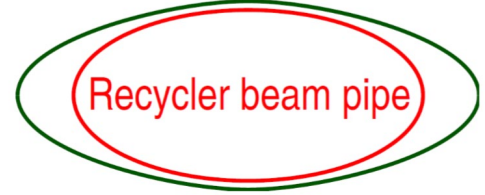

\section{Electron Cloud Instability:}
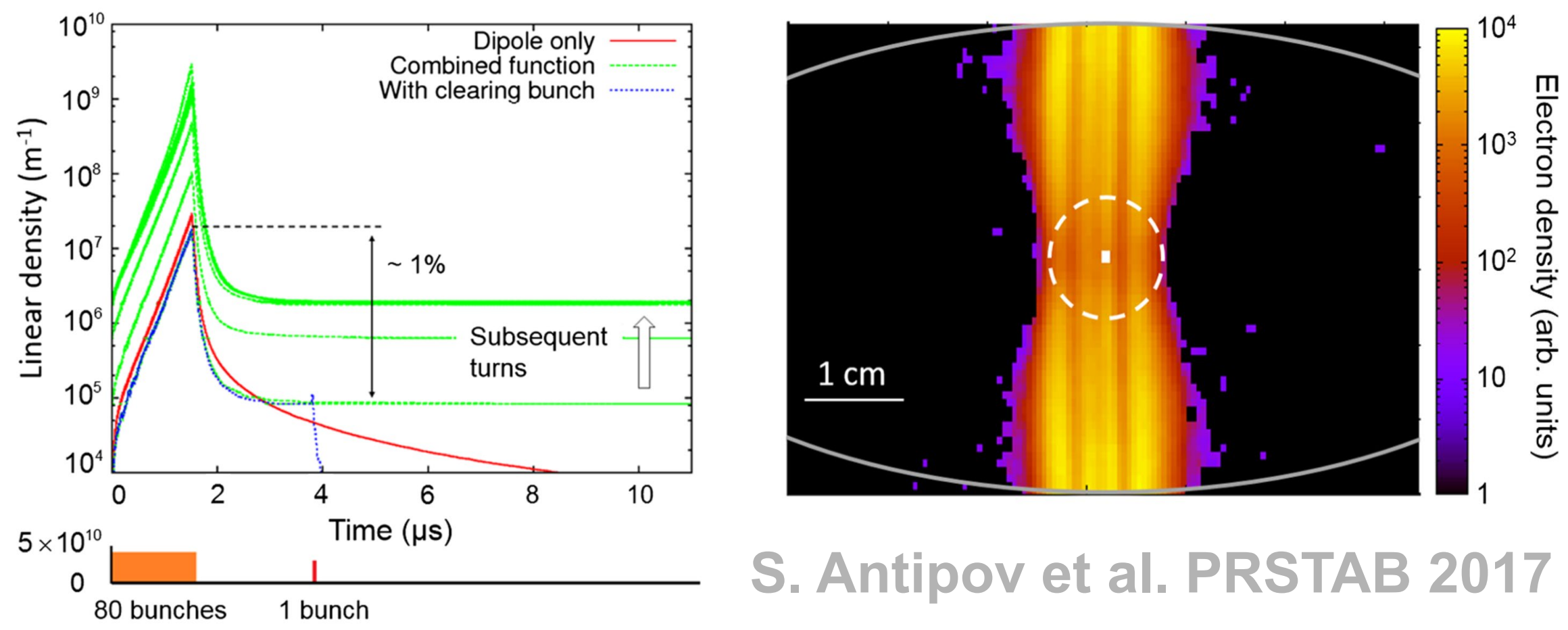

S. Antipov et al. PRSTAB 2017 


\section{Main Injector Intensity Upgrade}

Aperture and Space-charge Tune-spread

- $4.3 \mathrm{~mm}$ mrad aperture restriction for Ml

- $40 \mathrm{~mm}$ mrad normalized admittance at $8 \mathrm{Gev}$ or $50 \mathrm{~mm}$ mrad at $10 \mathrm{GeV}$.

- Alleviated by injecting into Ml at higher energy.

- Lattice correction of harmonic betatron resonances.

Reactive power needed to drive RF cavities

- For PIP-II we can add new power amplifiers to existing RF cavities.

- For 2.4 MW, we need to replace RF cavities and PAs.

$\mathrm{Y}_{\mathrm{T}}$-Jump for MI Transition Crossing

High-Power Neutrino Target 


\section{Main Injector Intensity Upgrade}

\section{$\mathrm{Y}_{\mathrm{T}}$-Jump for MI Transition Crossing}

- Pulsed quads for $\mathrm{Y}_{T}$-jump to be installed for PIP-II.

- For 2.4 MW, consider going from negative to positive chromaticity at transition crossing.
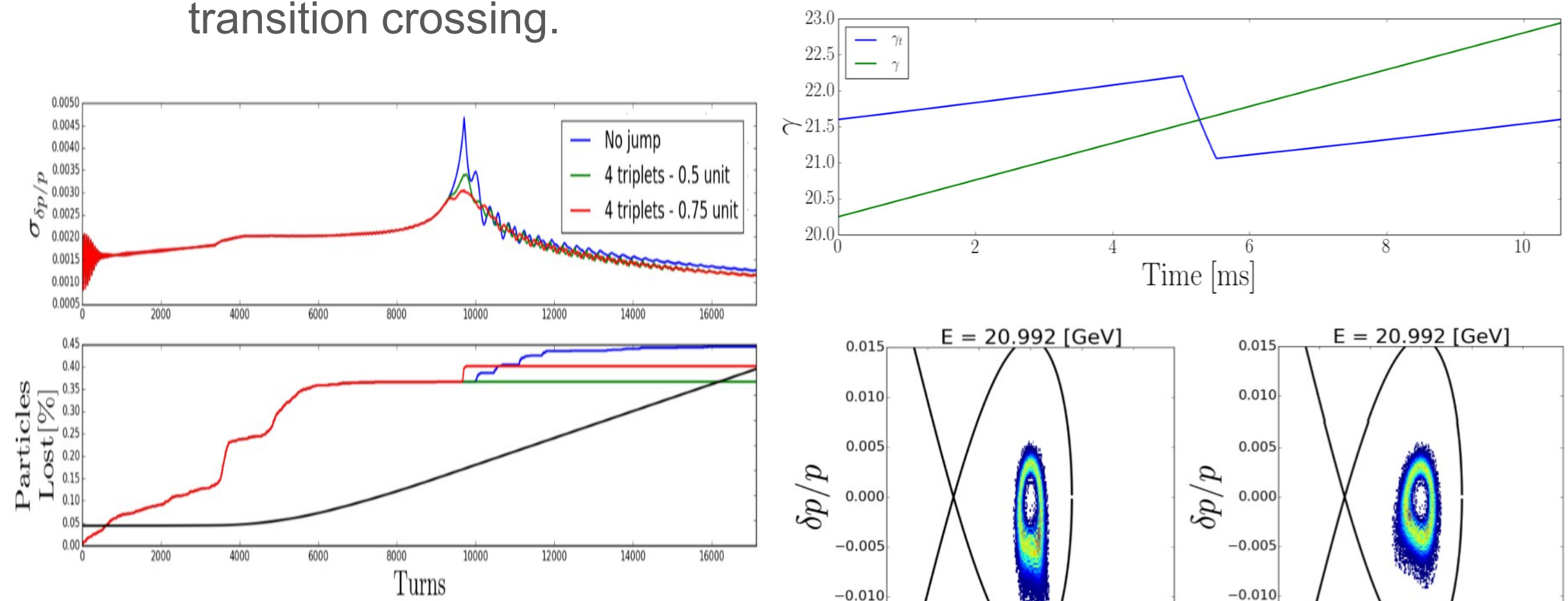

R. Ainsworth et al. IPAC 2017
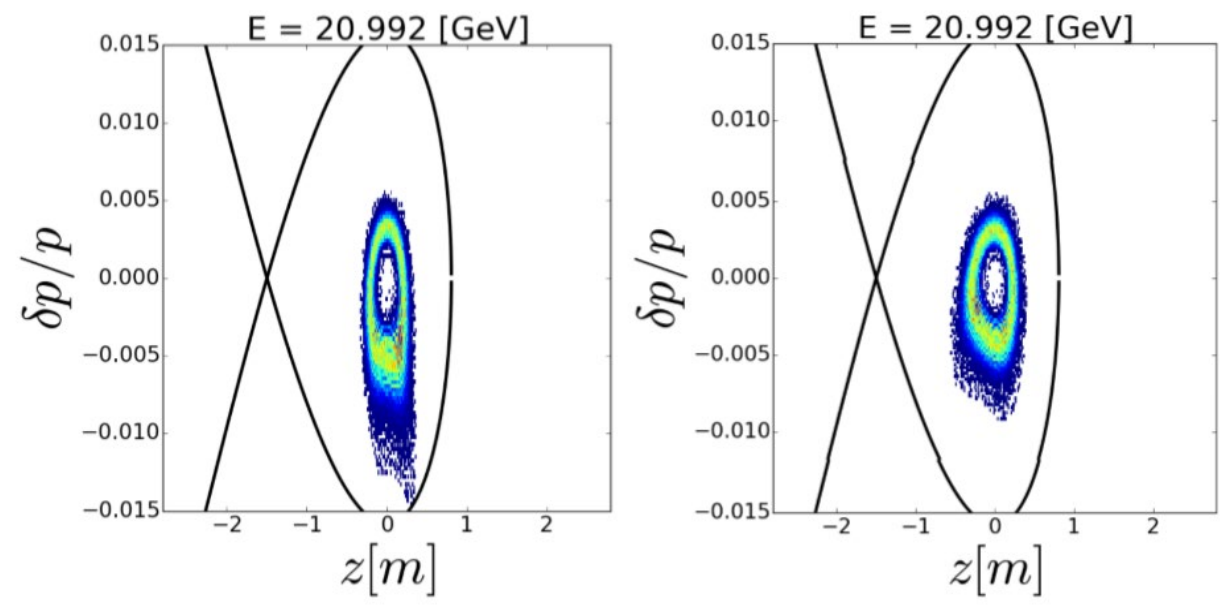

茎 Fermilab

23 Jeffrey Eldred | RCS for Multi-MW Facility at Fermilab 


\section{Main Injector Intensity Upgrade}

\section{High-Power Neutrino Target}

- Detailed design of 2.3 MW Be target for neutrino beams.

- Active area of R\&D development - shock test, radiation damage, target design, flux optimization etc.
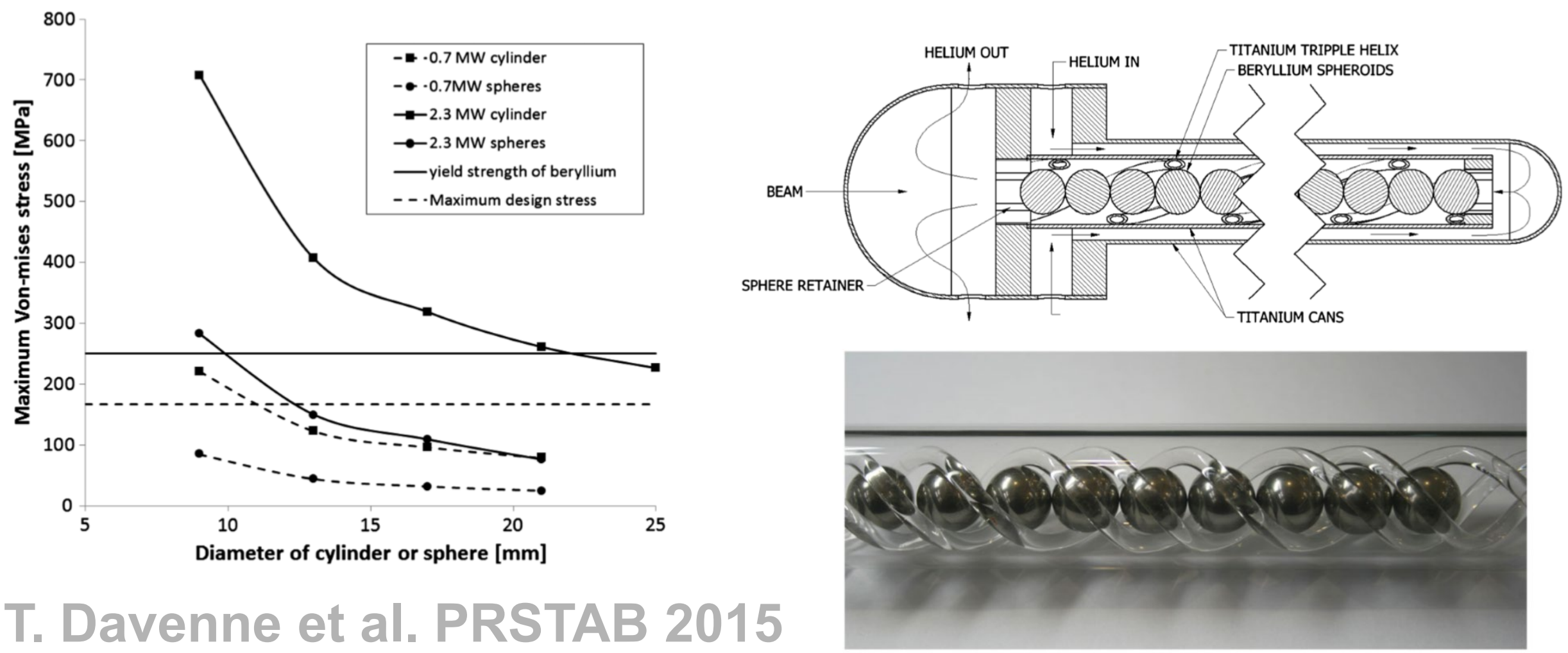

T. Davenne et al. PRSTAB 2015 


\section{Beyond 2.4 MW}




\section{Options for Intensity Beyond 2.4 MW}

If future physics experiments need even higher power proton beams at $120 \mathrm{GeV}$, how can that be accomplished?

\section{Options:}

1) Better performance from the new RCS

- IOTA technologies such as integrable optics or electron lenses?

2) Extend PIP-II linac to raise RCS injection energy

- An upgrade to a 1.4 GeV Linac could double the RCS intensity.

3) Reduce MI ramp time

- Add magnet power supplies, RF stations, replace RF cavities

4) Use 4+5 Slip-Stacking with a harmonic cavity

- Use harmonic RF to improve stability and bring RF buckets closer.

- Stack in the Main Injector, $\mathbf{+ 5 0 \%}$ beam power

- Or build a new Recycler, $\mathbf{+ 8 0} \%$ beam power. 


\section{Conclusions}

1. A new RCS can quadruple intensity per unit length by avoiding transition crossing, reducing betas, increasing periodicity, and improving lattice correction.

2. To fill the RCS at $5 \mathrm{~mA}$, the PIP-II Linac would require a power amplifier upgrade.

3. It may be preferable to inject directly into the $\mathbf{M I}$ at $\mathbf{1 0}-\mathbf{G e V}$ and eliminate the use of the Recycler.

4. At 2.4 MW, the Main Injector would need to new RF cavities and PAs to provide the reactive power for the beam loading.

5. An RCS also opens up good options beyond 2.4 MW. 


\section{Backup}

\section{Fermilab}




\section{Harmonic Slip-stacking}

\section{Eldred, Zwaska PRAB 2016}
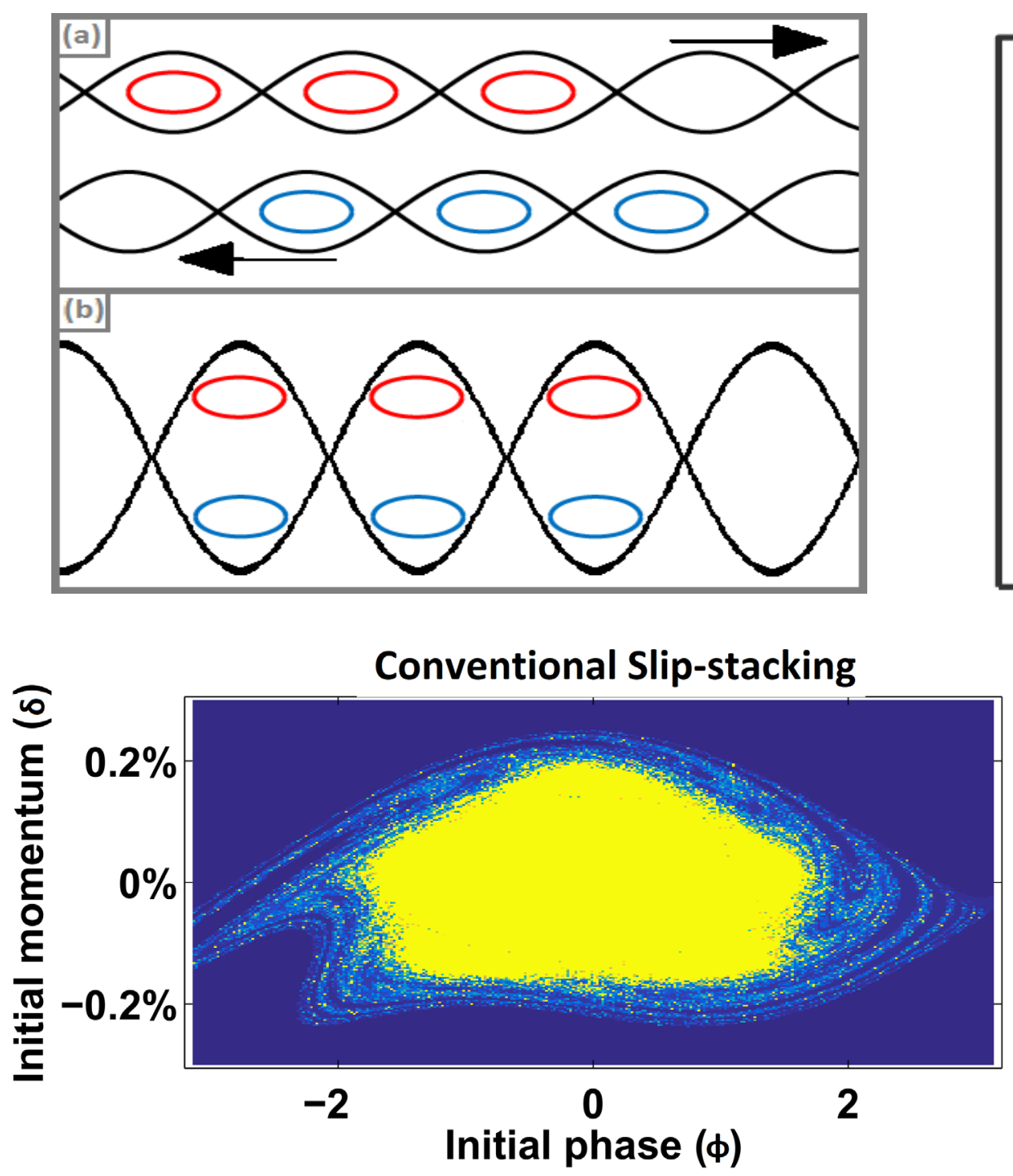
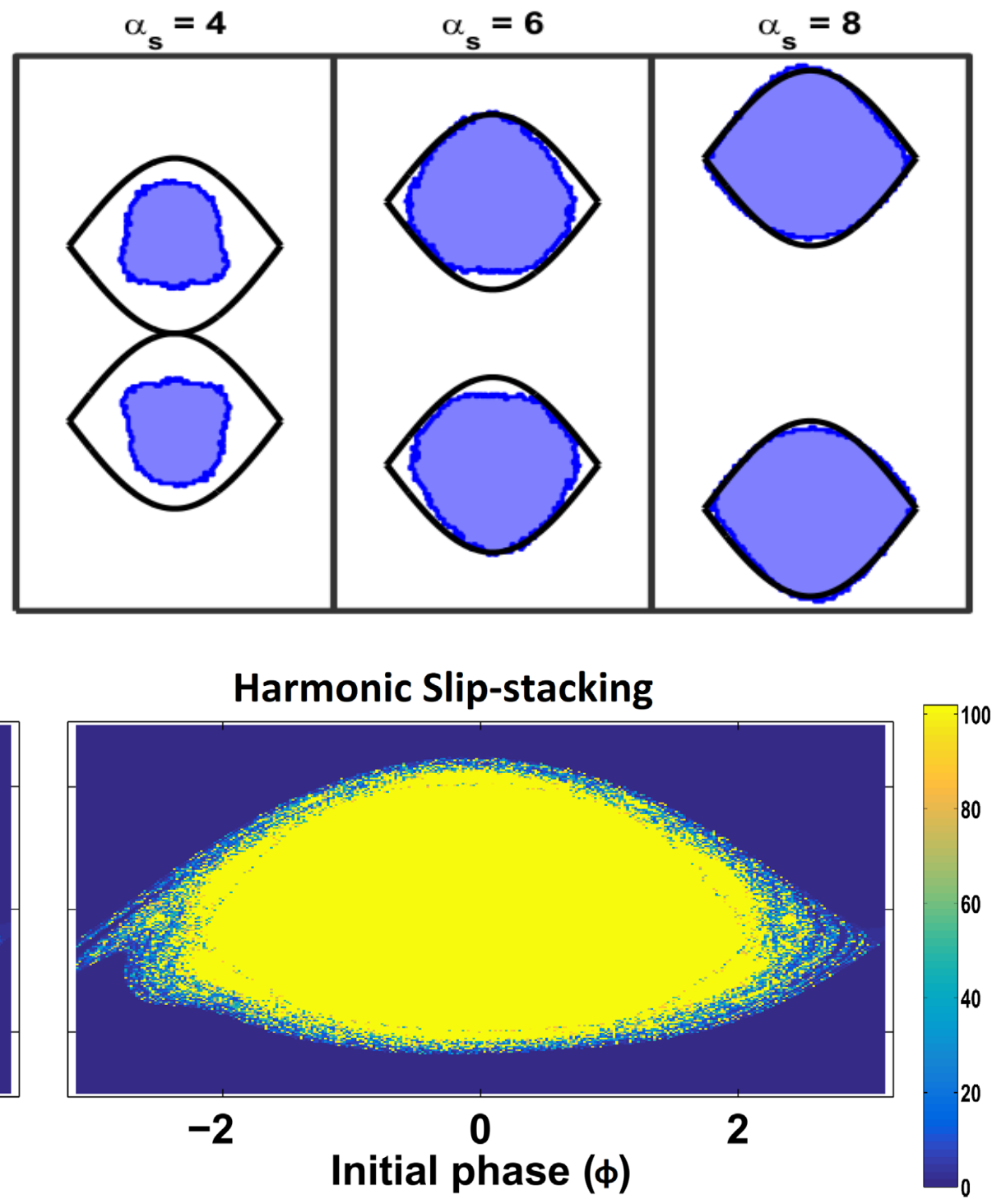

중 Fermilab 


\section{Backup \\ Lebedev RCS Design}




\section{Rapid Cycling Synchrotron for PIP-Il+}

- New RCS is aimed to support 2.4 MW beam power to LBNE

- Its $20 \mathrm{~Hz}$ rep. rate corresponds to $760 \mathrm{~kW}$ beam power of RCS beam and will be greatly supportive to $8 \mathrm{GeV}$ program

- The ring high periodicity suppresses the resonances driven by beam space charge

- FODO optics is chosen

- Simple and uniform through the ring

- Zero dispersion in straights

- Betatron phase advances per cell are less than 90 deg.

- No transition crossing

- Reduction of B field in dipoles reduces heating of vacuum chamber by eddy currents

- Circumference of RCS is larger than Booster circumference (1/6 of MI circumference instead of $1 / 7$ )

- Larger betatron tunes increase number of dipoles and quads and reduce percentage of orbit taken by dipoles. It yields that Booster: $B_{\max }=7.26 \mathrm{kG} \Rightarrow \mathrm{RCS}: \mathrm{B}_{\max }=8.09 \mathrm{kG}$ (in spite of larger circumf.) 


\section{RCS Beam Optics}

- All dipoles and Quads are connected serially

- Trim quadrupoles located in each corrector pack near each quad correct discrepancy between quad and dipole fields and set tunes and optics

- Resonance circuits tune the ramp frequency to $20 \mathrm{~Hz}$

- Apertures are set by acceptance of MI

Parameters of beam optics

\begin{tabular}{|l|c|}
\hline Circumference & $553.24 \mathrm{~m}$ \\
\hline Number of super periods & 10 \\
\hline Number of cells per super period & 7 \\
\hline Betatron tunes, Qx/Qy & $13.81 / 13.80$ \\
\hline Phase advances per cell & $0.1973 / 0.1971$ \\
\hline Momentum compaction & 0.007783 \\
\hline Transition energy (kin.) & $9.697 \mathrm{GeV}$ \\
\hline Natural chromaticities, $\xi x, \xi y$ & $-15.6 /-15.7$ \\
\hline Acceptance (geom.) & $57 \mathrm{~mm} \mathrm{mrad}$ \\
\hline
\end{tabular}




\section{Dispersions, Beta-functions and Betatron Phase Advances}

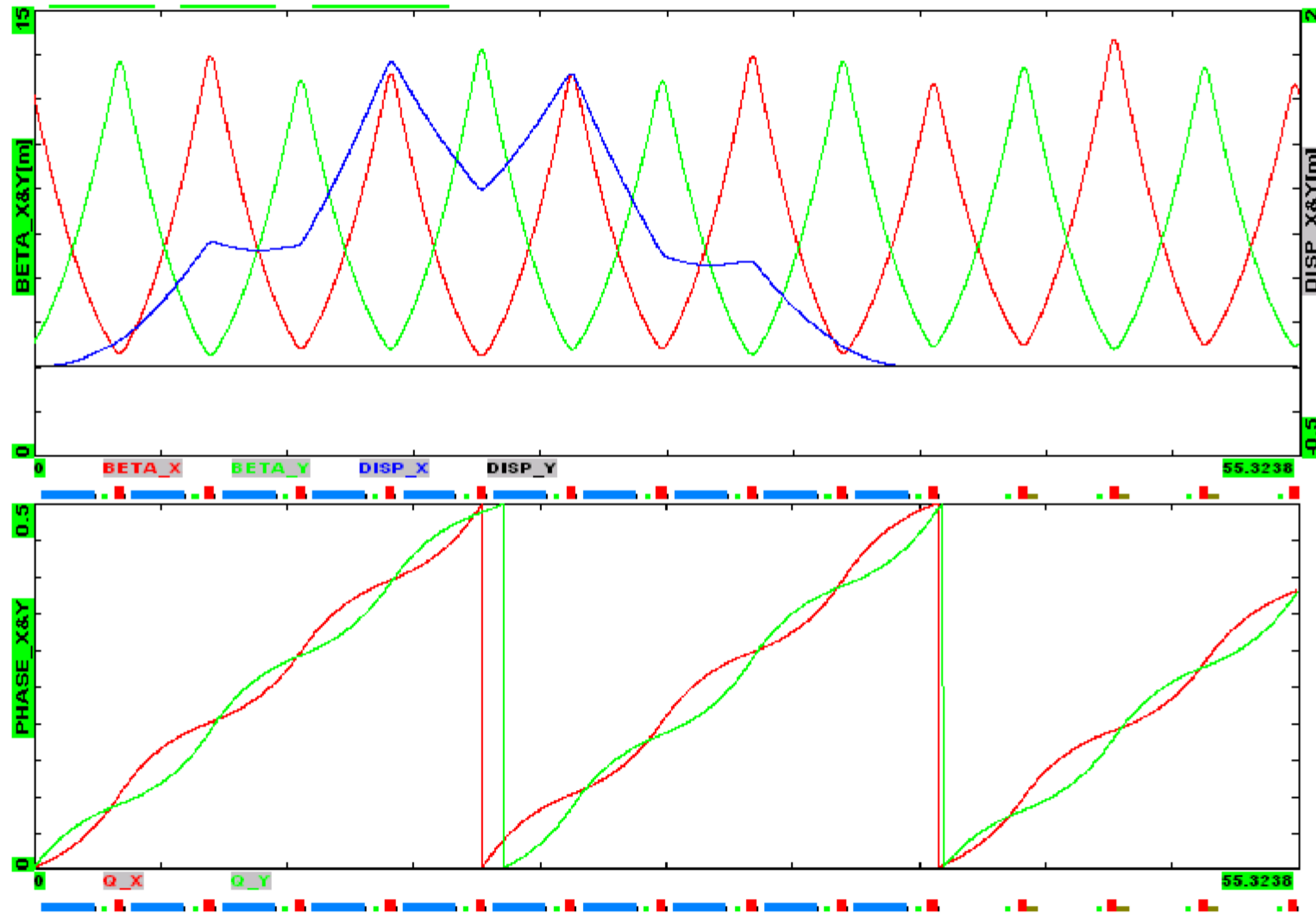

Fermilab Workshop on Megawatt Rings \& IOTA/FAST Collaboration, Valeri Lebedev, May 7-10, 2018, Fermilab 


\section{Acceptances and RMS emittances}

- Acceptance of RCS is matched to the acceptance of MI determined by the vacuum chamber in dipoles (other aperture limitations in $M I$ are not

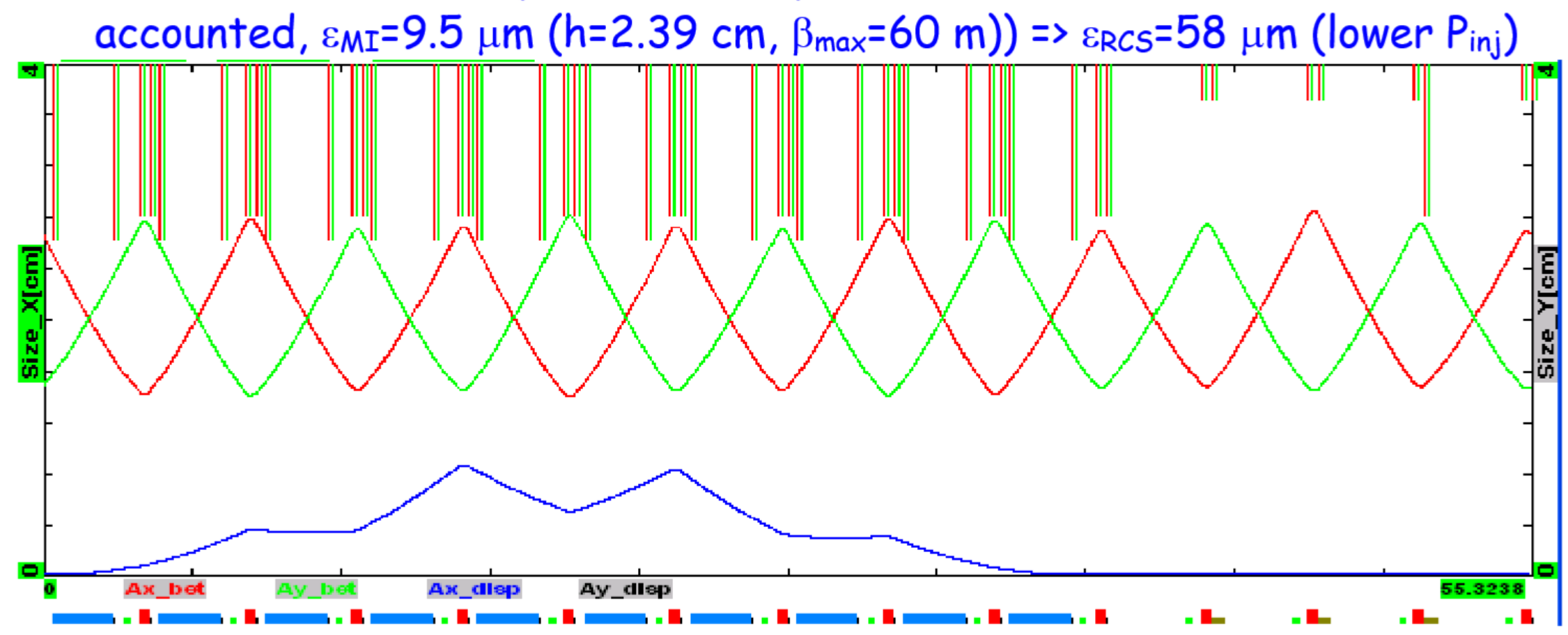

Beam envelopes at the acceptance ( $\mathcal{E}=58 \mathrm{~mm} \mathrm{mrad}$ ) and maximum $\Delta p / p=5 \cdot 10^{-3}$

- Accounting allowances for vacuum chamber $(2 \mathrm{~mm})$ we obtain apertures: in dipoles $r=28 \mathrm{~mm}$ and in arc quads $r=30 \mathrm{~mm}$

- Steering errors are already accounted in MI aperture

- Quads in straights have larger aperture to accommodate injection and extraction 


\section{Parameters of Magnets}

\begin{tabular}{|l|c|}
\hline \multicolumn{2}{|c|}{ Dipoles } \\
\hline Number of dipoles & 100 \\
\hline Dipole length & $2.302 \mathrm{~m}$ \\
\hline Dipole magnetic field at $8 \mathrm{GeV}$ & $8.09 \mathrm{kG}$ \\
\hline Gap & $56 \mathrm{~mm}$ \\
\hline \multicolumn{1}{|c|}{ Low aperture (located in arcs) quads } \\
\hline Number of quads & 110 \\
\hline Quad length & $40 \mathrm{~cm}$ \\
\hline Quad gradient at $8 \mathrm{GeV}$ & $2.3 \mathrm{kG} / \mathrm{cm}$ \\
\hline Aperture ( $\varnothing$ ) & $60 \mathrm{~mm}$ \\
\hline Large aperture (located in straights) quads \\
\hline Number of quads & 30 \\
\hline Quad length & $50 \mathrm{~cm}$ \\
\hline Quad gradient at $8 \mathrm{GeV}$ & $1.84 \mathrm{kG} / \mathrm{cm}$ \\
\hline Aperture ( $\varnothing$ ) & $100 \mathrm{~mm}$ \\
\hline Number of quads & 30 \\
\hline
\end{tabular}




\section{Beam Acceleration in RCS}
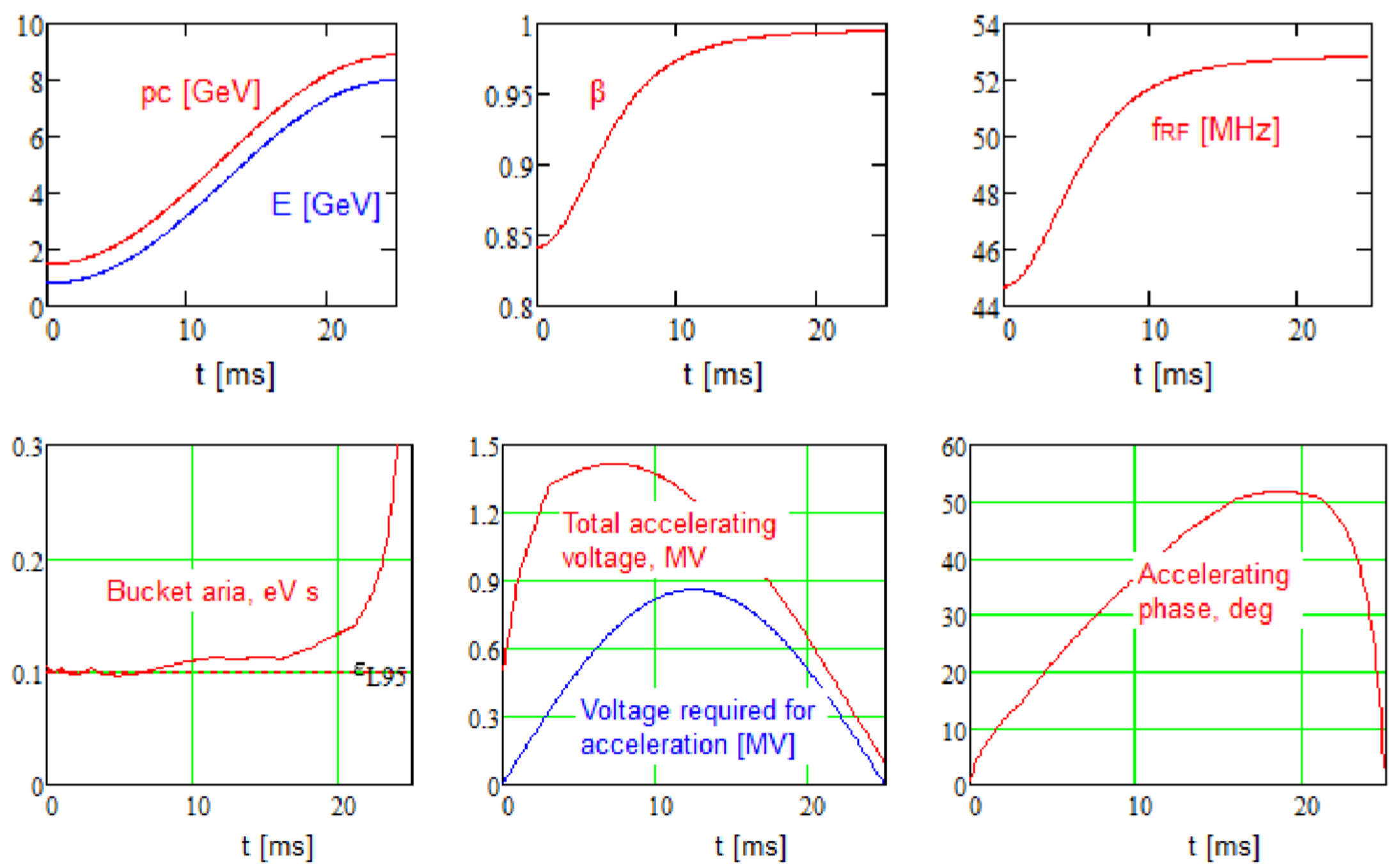


\section{Beam Acceleration in RCS $\left(P_{M I}=2.4 M W\right)$}

- Beam power at $8 \mathrm{GeV}-770 \mathrm{~kW}$

- 20 cavities @ 75 $\mathrm{kV}$
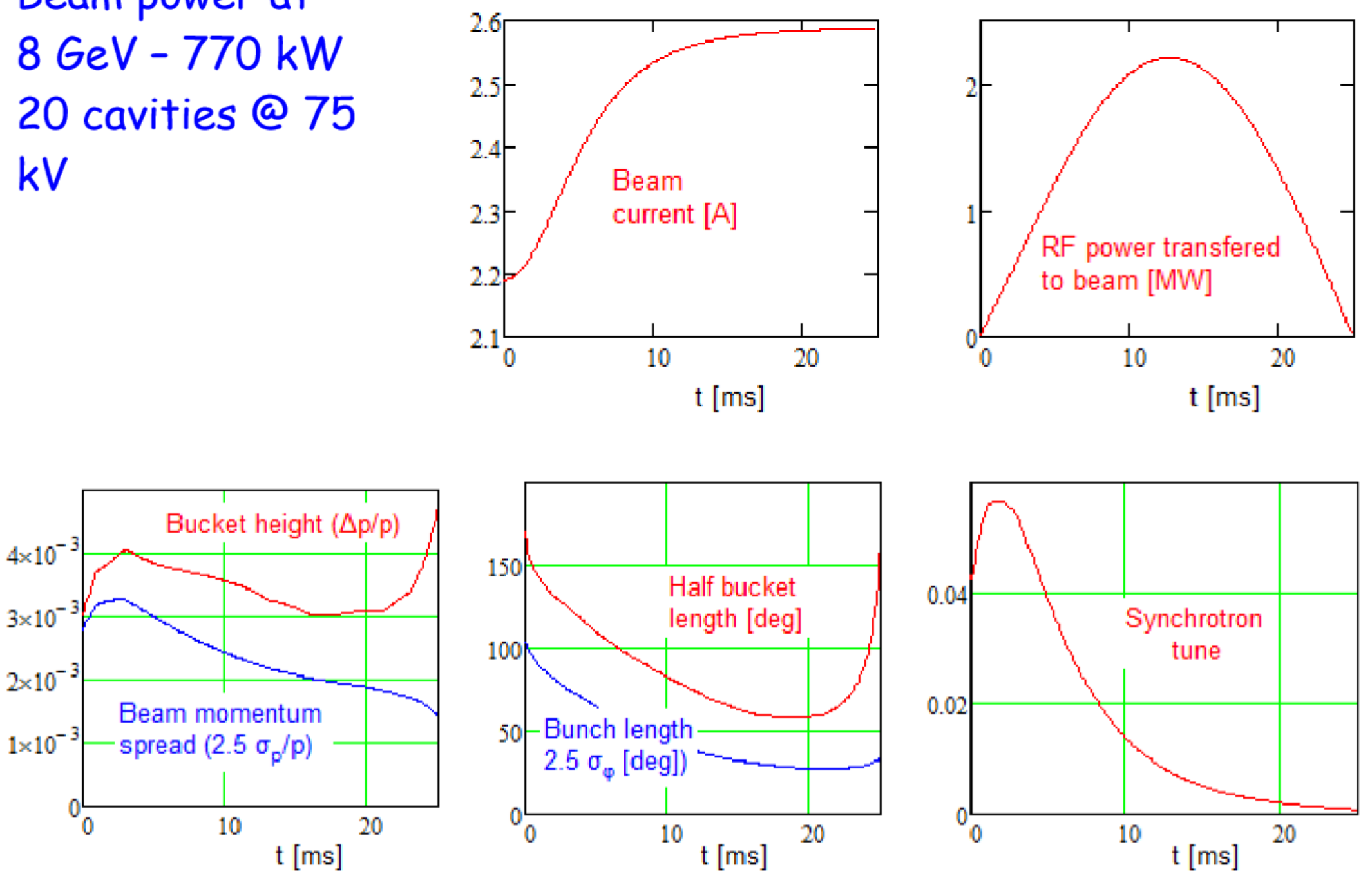


\section{Space Charge Tune Shifts for Supersymmetric RCS}

- RCS optics is built from 10 identical periods

- If periodicity is sufficiently accurate $(\Delta \beta / \beta<5 \%)$ then the space charge tune shifts have to be accounted for 1 period:

$\Rightarrow \Delta v_{x, y} \approx 0.062$

- Realistic simulations are required

- Experimental prove should come from PIP-I+ and IOTA

- To mitigate SC effects was chosen $71^{\circ}\left(<90^{\circ}\right)$

- Phase advance per period ( 1.38) is far enough

- Additional linac energy
- Phase advance per cell from 4-th resonance

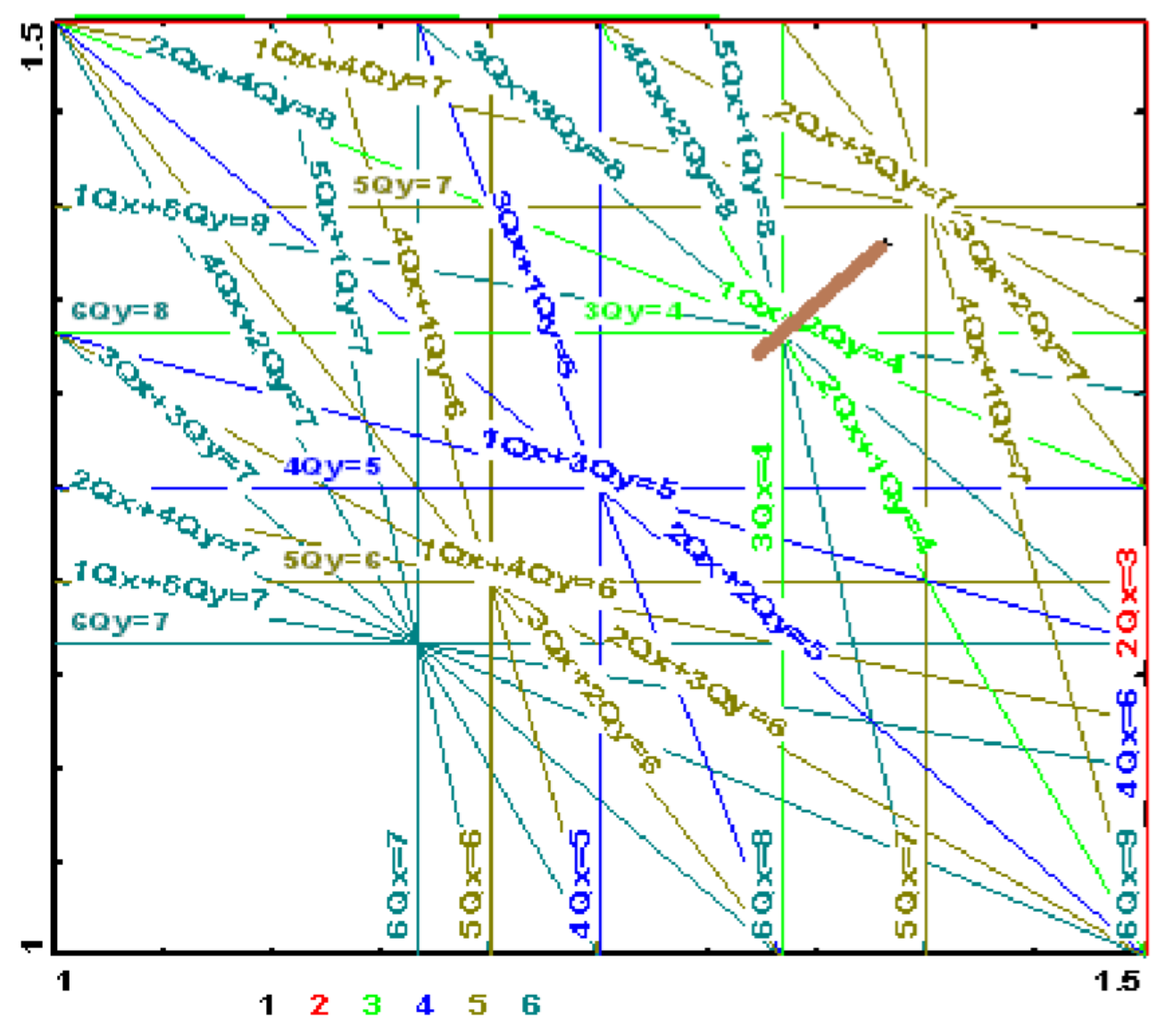

increase may require to mitigate the space charge 


\section{Injection}

- To keep supersymmetry of the ring 3 central quads and nearby corrector packs in each straight will have an increased aperture

- Sextupoles are not required in the straights

- Strip injection through foil (similar to ICD-2 proposal) will be used

- KV distribution painting in both transverse planes

- Peak foil temperature $1300 \mathrm{~K}^{\circ}$

- During 1100 turns injection the bending field is changed by $2.9 \%$.

- It can be compensated by correctors. 22 of $40 \mathrm{~A}$ is used if Booster like correctors are used

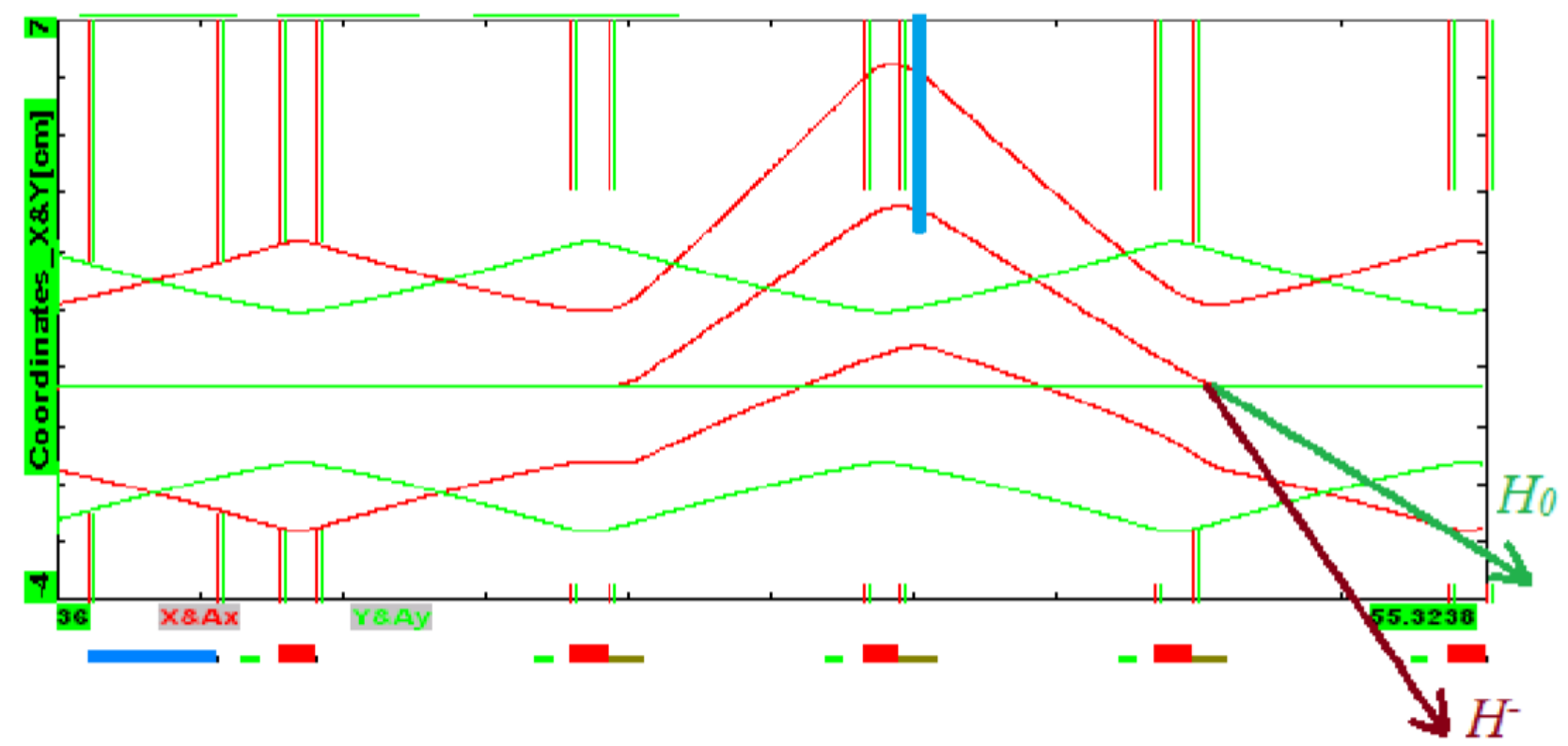




\section{Extraction}

- Extraction with vertical kicker (200 cm and $770 \mathrm{G})$ and Lambertson septum (200 cm and $13 \mathrm{kG}$ )

- Orbit distortion at may reduce required kicker strength

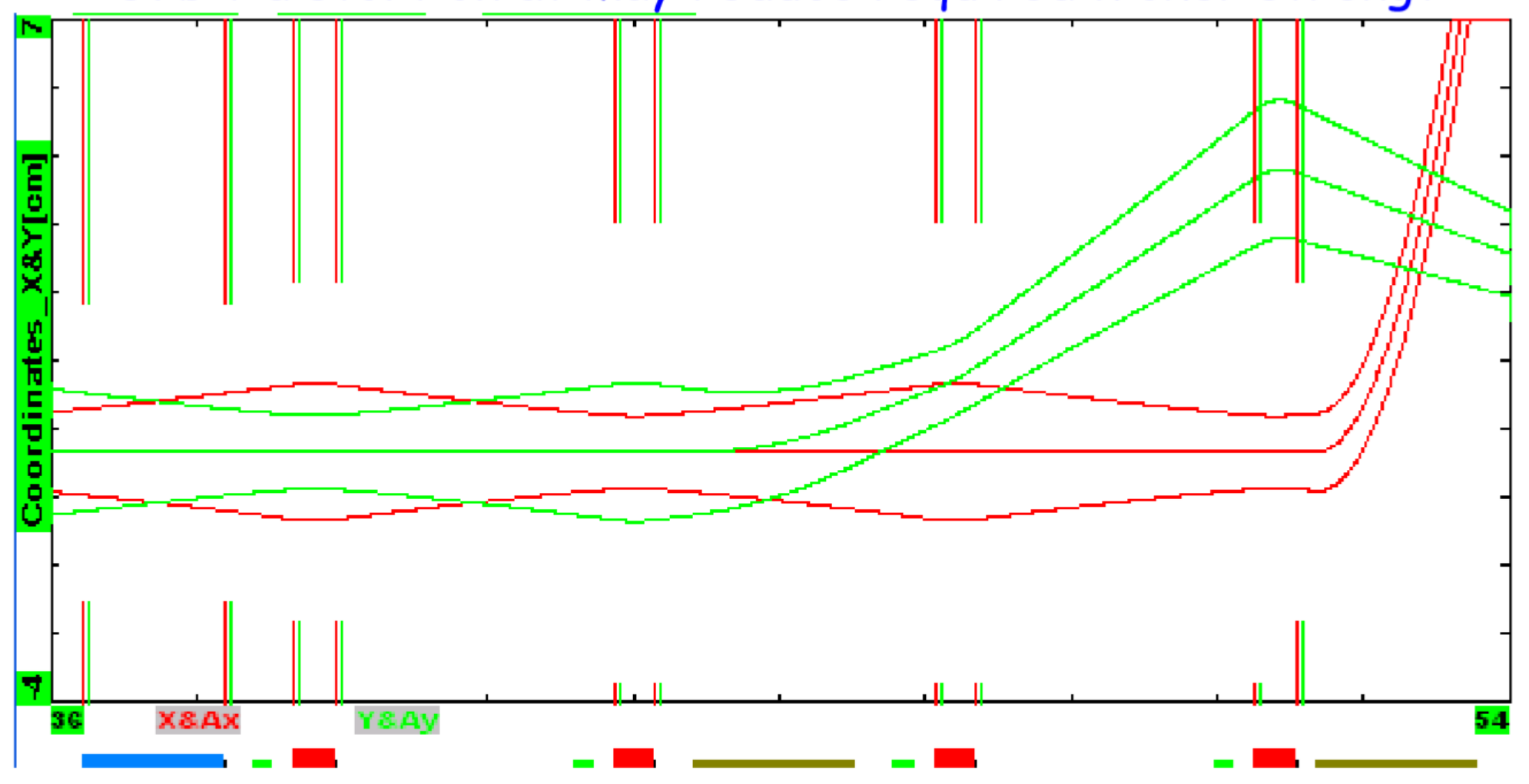

